\title{
FEASIBILITY OF TRANSHEPATIC ULTRASONIC EVALUATION OF INFERIOR VENA CAVA DIAMETER TO GUIDE FLUID MANAGEMENT IN COMPARISON WITH ANTERIOR APPROACH
}

\author{
Nawfal Ali Almubarak ${ }^{@}$, Mohammad Ali Hussein* \& Mazin Adnan \\ Abbas $^{\#}$ \\ ${ }^{\circledR}$ MB,ChB, Diploma of Anesthesiology, FICMS, Consultant of Anesthesiology and intensive care, \\ Assistant Professor, Basrah College of Medicine, Head of CABMS A \& IC Basrah Center, "MB,ChB, \\ Arabic Board Trainee of Anesthesiology, Al-Sadr Teaching Hospital, Basrah, Iraq. ${ }^{*}$ MB,ChB, FICMS \\ Anesth. Senior Anesthesiologist, Al-Sadr Teaching Hospital, Basrah, Iraq.
}

\begin{abstract}
Non-invasive estimation of right atrial pressure from the inspiratory collapse of the inferior vena cava using ultrasound machines is nowadays promising with the increasing availability of these machines in emergency departments, critical care units and in operating theaters. The learning curve of these tools is steep and they are becoming less expensive and give quick, noninvasive, dynamic clue to the preload status.

The aim of this study is to evaluate the transhepatic inferior vena cava (IVC) diameter by noninvasive sonographic imaging and compare it with that measured by subxiphoid approach to reveal the degree of agreement between them.

This is a prospective study, done in a tertiary care referral hospital at intensive care unit in AlSadr Teaching Hospital over a period of four months from 1st February 2017 to 5th of June 2017. Eighty shocked patients were included for assessment of volume status. Bedside ultrasound images were obtained with the patient in supine position to determine the dimensions and collapsibility of IVC. Evaluation of each patient included the standard anterior subxiphoid IVC assessment and lateral transhepatic assessment of IVC by ultrasound using liver as an acoustic window in mid-axillary line. The IVC diameter was measured $2 \mathrm{~cm}$ caudal to the hepatic vein-IVC junction, or approximately $3-4 \mathrm{~cm}$ caudal to the junction of IVC and the right atrium. Then IVC maximal diameter, IVC minimal diameter and collapsibility index were calculated for both approaches. A comparison between these data was made.

A total of eighty patients were included, $31(37.5 \%)$ were females and $49(62.5 \%)$ were males. Mean age was $45.81 \pm 14.89$ years. The results showed that there is no statistically significant difference between transhepatic and subxiphoid approaches in regard to IVC diameter and its collapsibility. In conclusion, transhepatic lateral view of IVC provides a good alternative when subxiphoid anterior view cannot be obtained to guide fluid management in shocked patients.
\end{abstract}

\section{Introduction}

Sonography has traditionally been used $\mathcal{O}$ to assess the anatomic abnormalities. However, its value in evaluating physiologic characteristics has recently been recognized particularly in the care of patients with hypovolemia. As the use of point-of-care sonography grows in critical care and emergency medicine, noninvasive assessment of intravascular volume status is increasingly being used to guide therapy of the critically ill ${ }^{1}$. Severe hypotension due to hypovolemia or sepsis is continuously challenging the management of hospitalized patients by shock itself or by multi-organ failure that is caused by inadequate tissue perfusion.

Fluid replacement without an accurate guide with a possibility of either underestimation or overestimation of fluid replacement gives a poor outcome in regard to morbidity and mortality in addition to the financial costs of prolonged hospital stay. Rapid and accurate assessment of volume status may 
improve outcome by guiding a proper intervention ${ }^{2}$.

Pulmonary artery and central venous pressure catheters that are the standards for decades to give physiologic data about right atrial pressure are time-consuming, invasive and have intrinsic risks. Central venous pressure (CVP) has long been used to guide fluid management; however, recent researches failed to prove that its data correlate with the effective intravascular volume ${ }^{3}$.

Pulmonary artery, central venous catheters or even peripherally inserted central catheter (PICC) have a list of inherited complications ${ }^{4}$. Complications such as arrhythmias, cardiac chamber injury, neural injury, pneumothorax, hemothorax, hematoma, infection, thrombosis and air embolism may occur with catheter placement ${ }^{5,6}$.

Noninvasive determination of right atrial pressure (RAP) from the inspiratory collapse of IVC using ultrasound machine is nowadays promising and more easily to be obtained in emergency departments, critical care units and in operating theaters. The learning curve of these rewarding tools is steep and they are relatively less expensive and provide quick, non-invasive and dynamic clue to preload status and fluid responsiveness. Recent researches have proved that they are acceptably reliable tools for all nonexpert clinical specialties, being able of determining intravascular volume status in severe hypotension and shock, a rapid bedside ultrasonic examination can guide medical management of critically ill patients ${ }^{7}$.

The IVC is a compliant, highly collapsible vessel that distends and collapses according to volume changes. Although the absolute IVC size has wide variety among individuals and may not by itself be diagnostic, the maximal IVC diameter has been shown to be lower in patients with hypovolemia ${ }^{8}$. There is no correlation between its diameter or collapsibility index, regarding to age, sex or body surface area ${ }^{9}$.

A better indicator of intravascular volume is collapsibility of the IVC. With a decrease in intra-thoracic pressure during inspiration in spontaneous breathing, venous blood is pulled into the right atrium. Consequently causes a decrease in IVC diameter. With expiration, IVC diameter increases (respirophasic variability). The IVC collapsibility caval index (CI) is defined as the difference between the maximal (expiratory) and minimal (inspiratory) IVC diameters divided by the maximal diameter. The caval index is used in spontaneously breathing patients to indirectly have a clue about RAP $^{10,11}$. The IVC-CI can provide a guide for noninvasive intravascular volume status assessment of critically-ill patients $^{10,12,13}$.

With deep breathing, there will be more accurate estimation of volume status; however, measurements taken during normal respiration are reasonably accurate as well ${ }^{12}$. The IVC respiratory collapse on sonography is easily imaged and can be used to roughly estimate RAP. The IVCCI of greater than or equal to $50 \%$ indicates RAP of less than $10 \mathrm{mmHg}$, and CI less than $50 \%$ indicates RAP greater than or equal to $10 \mathrm{mmHg}$ as shown in Table $\mathrm{I}^{10}$.

Table I: Correlation between IVC size, collapsibility and right atrial pressure, representing low, intermediate, and high RAP $^{10}$.

\begin{tabular}{|l|l|l|l|}
\hline & IVC Size & \% Collapse & RA Pressure \\
\hline Low & $<2.1 \mathrm{~cm}$ Normal & $>50 \%$ Normal & $3 \mathrm{mmHg}$ \\
\hline \multirow{2}{*}{ Intermediate } & $<2.1 \mathrm{~cm}$ Normal & $<50 \%$ Abnormal & \multirow{2}{*}{$8 \mathrm{mmHg}$} \\
\cline { 2 - 3 } & $>2.1 \mathrm{~cm}$ Abnormal & $>50 \%$ Normal & \\
\hline High & $>2.1 \mathrm{~cm}$ Abnormal & $<50 \%$ Abnormal & $15 \mathrm{mmHg}$ \\
\hline
\end{tabular}


A CI greater than $50 \%$ suggests a low volume status, especially in combination with a small IVC diameter. Conversely, a low CI with a large IVC diameter suggests a high volume status ${ }^{10}$.

To predict fluid responsiveness in critical conditions, dynamic parameters (stroke volume variation, pulse pressure variation, leg raising and IVC variation) should be used preferentially rather than static parameters (CVP, pulmonary capillary wedge pressure and physical examination); these parameters can be explored with a good accuracy by ultrasonic IVC imaging during respiratory cycles $^{14}$.

Recent guidelines from the American Society of Echocardiography support the general use of IVC size and collapsibility in assessment of volume status ${ }^{15}$.

Several studies have suggested the use of specific parameters for maximal IVC diameter and CI to predict volume status $^{10,12}$. One of these studies, suggests that using $2 \mathrm{~cm}$ as the cut-off for the maximal IVC diameter gives acceptable sensitivity and specificity in predicting elevated right atrial pressure ${ }^{12}$. Guidelines from the American Society of Echocardiography recommend examining it just distal to the hepatic veins, which lies approximately $3-4 \mathrm{~cm}$ from the right atrium $^{15}$.

Emergency physicians' ultrasound measurement of IVC diameter has a high degree of inter-rater reliability. The IVC percent collapse by visual estimation or based on caliber measurements has low, but still moderate to good reliability ${ }^{16}$.

The IVC size does not predict RAP in patient receiving mechanical ventilation in the same way it does in spontaneous breathing ${ }^{17}$. Mechanical ventilation (positive pressure ventilation) reverses the hemodynamics of venous return during the respiratory cycle. With inspiration in mechanical ventilation, intra-thoracic pressure increases, which impedes blood flow from the IVC to the right atrium. During expiration, intra-thoracic pressure is lower and venous return increases. When there is volume depletion, right atrium and IVC becomes more compliant, and there is more increase in IVC size than in euvolemia. The variation of the IVC in mechanically ventilated patients, which is known as the IVC distensibility index, is the difference between the maximum and minimum IVC diameters divided by the minimum diameter. In contrast to $\mathrm{CI}$, which indicates volume status in accordance to IVC collapse during spontaneous breathing, the distensibility index has been used to assess preload dependence and predict fluid responsiveness such that the absence of respiratory variation suggests that volume expansion is unlikely to be effective anymore, but this is to be done under full relaxation and with a tidal volume of $8 \mathrm{ml} / \mathrm{kg}^{18,19}$.

Fluid responsiveness is an emerging and important concept in critical care in an effort to avoid over estimated fluid administration when a fluid challenge is unlikely to improve hemodynamics and organ perfusion. Fluid responsiveness (FR) is a measure of preload dependence defined as an increase in cardiac output secondary to volume expansion. Inadequate dilatation of the IVC after a fluid challenge is more sensitive than blood pressure for identification of hypovolemia ${ }^{20}$.

A low-frequency phased array transducer (3.5-5 MHz) is used. IVC lies in the retroperitoneal $2-3 \mathrm{~cm}$ to the right of the aorta. It is differentiated from aorta by its thinner walls, respiratory size variation, being connected to right atrium, its connection with hepatic vein and as less pulsatile than aorta. There is a considerable variation in the literature regarding the choice of site at which the IVC diameter should be measured. Studies mostly agree about a point $3-4 \mathrm{~cm}$ from $\mathrm{RA}^{10,12,19,21}$. Other studies measure the IVC just caudal to the junction with hepatic veins ${ }^{18,22,23}$.

The IVC diameter is measured 
perpendicular to the long axis of the IVC at end-expiration and end-inspiration. The finding of a small-diameter IVC with large inspiratory collapse (high CI) correlates with low volume states. This observation is seen in hypovolemic and distributive shock. Conversely, a large IVC with minimal collapse (low CI) suggests a high volume state such as cardiogenic or obstructive shock. IVC metrics do not change significantly based on patient position. For those patients who are unable to lay completely supine, a semi- upright measurement of the IVC for volume status may be an accurate alternative $^{24}$. The IVC diameter and inspiratory variability evaluation are also quantifiable and reliable approach to assess the hypervolemia associated with congestive cardiac failure. Normalization of inspiratory IVC collapse movement correlates with successful diuretic therapy and can be reliably used in its management ${ }^{25}$.

The M-mode Doppler can be used to graphically document the size and dynamic changes during respiratory cycle. It is recommended that $\mathrm{M}$ - mode sonography be used after adequately visualizing IVC variability in the B-mode to avoid inaccurate estimation of vessel size and collapse. Many studies tried to define normal IVC parameters such as size, collapsibility, and distensibility. Until then, assessment of IVC collapsibility is useful in the critically ill patient whose CI is in the extremes. Additionally, Caval sonography can be repeated during fluid resuscitation to follow up improvement of these parameters. With B-mode holds the most promise to deliver reliable measures of IVC diameter ${ }^{26}$.

The IVC diameter of more than $2.5 \mathrm{~cm}$ and minimal collapse $(<10 \%)$ correlates with raised right atrial pressure $>15 \mathrm{~mm}$ $\mathrm{Hg}^{27}$.

In a recent prospective study, a point-ofcare sonography evaluating cardiac contractility and IVC collapsibility in patients with suspected sepsis was shown to increase physician certainty and alter more than $50 \%$ of treatment plans. Pointof-care (POC) ultrasonography is per definition, a bedside examination performed and interpreted by the treating physician.

The development witnessed, in part, reflects equipment of increased quality, mobility and availability, the latter is a result of reduced costs.

POC ultrasonography is a safe and valuable tool, for intensivists. It has advantages in being repeatable and realtime examination tool that supplies images, which correlate directly to the symptoms or the clinically suspected diagnosis. In terms of airway, breathing, circulation (ABC-), it covers cardiac, pulmonary, abdominal and vascular ultrasound $^{28}$.

Over-estimation of intravascular volume may occur in conditions that impede flow to the right side of heart, including valvular abnormalities, pulmonary hypertension and congestive heart failure.

Interpretation of the physiologic characteristics of the IVC should be done in context with the patient's clinical scenario and adjunctive data ${ }^{29}$.

The position of scanning probe for IVC is not standardized. While the usual recommended location is subxiphoid transabdominal long axis view, there are limited data comparing this approach to another one which is transhepatic.

The aim of this study was to compare the size of IVC measured by transhepatic sonographic imaging with that measured by standard one, the subxiphoid anterior approach in hypovolemic, spontaneously breathing hypotensive patients.

\section{Patients and Methods}

This prospective study was done in a tertiary care referral hospital at intensive care unit of Al-Sadr Teaching Hospital in Basrah, Iraq.

Eighty hypotensive or shocked patients aged 18-80 years were included for 
assessment of volume status in an attempt to decide whether to continue on fluid administration or to shift into vasopressors. The study was performed over a period of four months from 1st February 2017 until 5th June 2017. Using world health organization (WHO) sample size calculator, a sample size of 80 was taken.

The sonographic evaluation was done by a final year anesthesia board trainee who completed a four week course run by a radiologist and an intensive care unit director with five years' experience in this evaluation, and then the reader developed an extended experience in the field for the following four months before the study. Bedside ultrasound images were obtained with the patient supine to measure the dimensions and determine collapsibility of IVC during deep spontaneous breathing.

Exclusion criteria included: Patients below the age of 18 years, pregnancy after the second trimester, congestive cardiac failure, raised intra-abdominal pressure, those on assisted ventilation or who cannot take deep breath, dysrrhythmias and tricuspid valve disease.

A portable M-Turbo ultrasound system B\FUJI FILM SonoSite machine, version of 06-2013\USA was used with the curvilinear probe of low frequency (2-5) MHZ transducer for all examinations.

First, ultrasound gel was applied to the anterior subxiphoid region where IVC assessment was done with inspiration ultrasonically $1-2 \mathrm{~cm}$ to the right of the midline by placing the probe horizontally on the patient's abdomen just below the xiphoid bone with the marker facing to the right of the patient. Once an appropriate subxiphoid view of IVC is obtained, probe is rotated 90 degree until the marker is pointing towards the head of the patient, at this point the IVC should be visualized in the longitudinal plane as it enters the right atrium at this position.

The IVC diameter was measured $1-2 \mathrm{~cm}$ caudal to the hepatic vein- IVC junction or approximately 3-4 $\mathrm{cm}$ from the junction of IVC with right atrium, this position was preferred as IVC collapsibility in the intra hepatic segment is not affected by the muscular activity of diaphragm. The maximum IVC diameter (IVCmax) was measured at end of expiration using the leading edge technique (inner edge to inner edge of the vessel wall). In addition, the minimum IVC diameter was measured at end of inspiration (IVCmin). Then calculated the IVC-CI (\%).

Then lateral transhepatic assessment of IVC using liver as an acoustic window in anterior to mid-axillary line was done, similar to the placement for evaluating Morison's pouch (hepato-renal angle), the marker should be pointing to the head of the patient.

By scanning more anteriorly and cephalic than the Morison's pouch view, the IVC can be visualized running longitudinally adjacent to the liver and crossing the diaphragm. Following the vessel along until it enters the right atrium allows confirmation that the IVC is being visualized and not the aorta that running parallel and posterior to it. In the same way as to measure IVC diameter in subxiphoid approach, the IVCmax and IVCmin were estimated and then calculated for the IVC-CI (\%).

In both of approaches, B-mode was used to visualize IVC by right angle position and clear view of the IVC with careful observations of the patient's breath cycle (end inspiration and end expiration) then freeze the image and estimate the diameters.

When patient have decreased IVC diameter and the percentage collapse is greater than $50 \%$, it means volume depletion. On the other hand, if IVC diameter is large with minimal collapse on inspiration, it means volume overload.

Data were collected from 80 patients that have been taken from the subxiphoid IVC diameters and its CI percentage and transhepatic mid axillary IVC diameters and its CI percentage then a comparison between these data was made. 
Statistical analysis: Pearson correlation coefficient was used to assess the significance between IVC-CI (\%) and the maximum and minimum IVC diameters in subxiphoid and mid axillary approaches. P-value $<0.05$ was considered strong positive correlation between IVCmax and IVCmin in both approaches as well as their IVC-CI (\%).

Three cases were dropped from the study due to a failure of visualization by anterior approach after recent laparotomy.

\section{Results}

Eighty patients who have been admitted from different departments to ICU were included. The mean age was $45.81 \pm 14.89$ years. The sample is composed of 31 (37.5\%) females and 49 (62.5\%) males.

Twenty four post-operative general surgical patients (30\%), 13 medical patients (16.3\%), 12 orthopedic patients (15\%), 19 neurosurgical patients (23.8\%), 7 obstetrical patients (8.8\%) and finally, 5 thoracic-vascular patients $(6.3 \%)$. This is revealed by Table II.

Table II: Prevalence of included cases.

\begin{tabular}{|l|l|l|}
\hline Cases & Frequency & Percent \\
\hline General surgery & 24 & 30.0 \\
\hline Internal medicine & 13 & 16.3 \\
\hline Orthopedic & 12 & 15.0 \\
\hline Neurosurgery & 19 & 23.8 \\
\hline Obstetric & 7 & 8.8 \\
\hline Vascular & 5 & 6.3 \\
\hline Total & 80 & 100.0 \\
\hline
\end{tabular}

The mean of minimum diameter in transhepatic approach was $0.78 \pm 0.47 \mathrm{~cm}$ while the mean in subxiphoid approach was $0.8 \pm 0.44 \mathrm{~cm}$ with $\mathrm{p}$-value 0.39 and the mean of maximum diameter in transhepatic approach was $1.6 \pm 0.54 \mathrm{~cm}$ while its mean in subxiphoid approach was $1.64 \pm 0.5 \mathrm{~cm}$ with p-value was 0.49 and the mean of CI was $54.3 \% \pm 15.2$ and $53.8 \% \pm 15$ in transhepatic approach and subxiphoid approach respectively with a p-value 0.116 .

According to these statistical results, none of the parameters showed significant statistical difference. This is presented in Table III.

Table III: Correlation between sub xiphoid and Transhepatic approaches

\begin{tabular}{|l|l|l|l|}
\hline Parameter & Approach 1 Mean \pm SD & Approach 2 Mean \pm SD & P value \\
\hline Min. & $0.786 \mathrm{~cm} \pm 0.478$ & $0.800 \mathrm{~cm} \pm 0.438$ & 0.393 \\
\hline Max. & $1.623 \mathrm{~cm} \pm 0.539$ & $1.640 \mathrm{~cm} \pm 0.489$ & 0.491 \\
\hline Caval index \% & $54.316 \mathrm{~cm} \pm 15.235$ & $53.789 \mathrm{~cm} \pm 14.954$ & 0.116 \\
\hline
\end{tabular}

Approach 1: transhepatic. Approach 2: subxiphoid. MIN: minimum size of IVC diameters. MAX: maximum size of IVC diameters. SD: Standard deviation.

\section{Discussion}

Over the last decade, many clinical studies have led to the validation of sonography for evaluation of many critical cases as a first rapid assessment of pulmonary, cardiovascular and airway parameters in addition to being a bedside tool for focused assessment sonography of trauma (FAST) exam.

These machines are becoming available in different hospital departments as their use for resuscitation measures have progressively developed among different medical specialties.

Standard measurements of preload for fluid management in the near past included mainly central venous pressure 
or pulmonary artery occlusion pressure.

The Surviving Sepsis Campaign guidelines still recommend assessing hypovolemia and fluid therapy by using CVP, to be between 8 and $12 \mathrm{mmHg}^{30}$.

Uses of ultrasound for assessing the IVC diameter which provides a good alternative tool to CVP because it has less cost, less time consuming, non-invasive and avoids the complications of CVP insertion like arterial puncture, pneumothorax, hemothorax, dysrrhythmias, cardiac chamber injury, neural injury, infection, thrombosis and chylothorax (in left sided approach). Central venous pressure is even regarded of limited sensitivity in some circumstances as they are static parameters $^{31-33}$.

Sonography also provides dynamic interpretation of volumic status and fluid responsiveness by assessment of IVC diameter change especially with spontaneous breathing based on heart-lung interactions (dynamic estimation) ${ }^{34,35}$.

In addition, it reflects volume status more closely than other parameters like blood pressure, pulse rate or diameter of aorta ${ }^{14}$. The use of ultrasonography for IVC assessment in mechanically ventilated patients gives different reliability among different studies and is not studied here as it needs more precise measurements and hence, experience ${ }^{36-38}$.

In addition to the evaluation of hypovolemia and patients with severe sepsis, it can aid evaluation of fluid overload and congestive cardiac failure $^{23,39,40}$. The IVC-CI in B mode has been used on anterior subxiphoid region and compared in our study to lateral transhepatic view. The M-mode is used to precisely and dynamically measure IVC diameter but it has not been used here because it needs experience and is difficult to be done in some circumstances, for example when patient is breathing forcefully; thus inexpert can get wrong angle.

Fluid responsiveness in this study was assessed by making serial measurements after fluid administration and followed up till hemodynamic stability achieved with normalization of blood pressure and tissue perfusion or a vasopressor/inotropic is initiated. Straight leg raising test adds to the reliability but could not be done in a number of multitrauma patients so it has been excluded from the parameters.

Many clinical and experimental studies have been done but no enough data in regard to the comparison between the two approaches. Yanagawa et al (2005), used IVC diameter in expiratory phase in the early diagnosis of hypovolemic shock by ultrasound for trauma patients ${ }^{41}$. In this study, the IVC diameter in expiratory phase for the diagnosis of hypovolemia was $9 \mathrm{~mm}$. The diameter $(7.7 \pm 0.3 \mathrm{~mm})$ measured in the shock group was significantly lower than $(13.4 \pm 0.7 \mathrm{~mm})$ measured in the control group.

Another study was performed by Sefidbakht et al (2007), in patients with trauma $^{42}$. In this study, IVC diameters in expiratory and inspiratory phase were (5.6 \pm 0.8 and $4 \pm 0.7 \mathrm{~mm}$ ) of the shock group were also significantly lower than those of the control group (11.9 \pm 2.2 and $9.6 \pm 2 \mathrm{~mm}$ ) and CI was higher.

These studies suggest that IVC diameters can be considered a modestly reliable indicator of hypovolemia, although blood pressure is within normal limits because of sympathetic activation.

Akilli et al (2010), compared the IVC diameter with other parameters during shock state such as heart rate, systolic blood pressure, diastolic blood pressure, shock index, urine output, serum lactate level and base excess level in patients at emergency department with hemorrhagic shock. They concluded that the IVC diameters in such patients were more valuable than other conventional shock parameters $^{43}$.

Fields et al (2011), reported that the correlation coefficient of the IVC was 0.81 (95\% CI, 0.67-0.89) for IVC diameter in expiratory phase and 
correlation coefficient was 0.77 (95\% CI, $0.62-0.87$ ) for IVC diameter in inspiratory phase. They also stated that the interrater reliability of measurement was increased from 0.49 to 0.81 after expiration and from 0.51 to 0.85 after inspiration at the end of the first 5 examinations. Their studies suggest that 15 ultrasound examinations may be necessary to obtain sufficient skill ${ }^{44}$.

Akkaya et al (2013), found that there was an increase in the interrater reliability of IVC diameter during inspiratory phase measurements after 10 examinations ${ }^{45}$.

The interrater variability comparison was not performed in this study but instead, the same reader compared the two approaches (and was blinded to) a standard CVP measurement as a reference to add to the reliability of personal experience.

A similar study was done to reveal agreement between IVC diameters in subxiphoid approach versus transhepatic one, it was performed by Kulkarni AP et al (2015), showed that IVC diameter variability evaluation gives a clue about fluid responsiveness equally in both approaches, although the study was applied on patients with mechanical ventilation support while this study selected those who are spontaneously breathing ${ }^{46}$. There are obstacles in assessing obese patients, patients with recent abdominal surgeries and those in whom IVC is difficult to be visualized by standard anterior subxiphoid view; the transhepatic view overcomes this by assessing the IVC using liver as an acoustic window.

Rian et al (2016), identified that the IVC predominantly collapses into a horizontal pattern, and that flat ratio can predict concordance. They have also determined that the transhepatic view is less sensitive in predicting fluid responsively as compared to the current standard subcostal view ${ }^{47}$. Although in our study there was no significant difference between the two approaches.

Results in this study demonstrated that in spontaneously breathing hospitalized patients in ICU, emergency departments or operating theater, sonographic determination of IVC diameter change with adequate depth of spontaneous breathing, seems to be a useful tool in the early approach to the shocked patients.

In conclusion, none of the parameters have shown a significant statistical difference between the two approaches in an attempt to indirectly assess volume status and guide fluid managements in shocked patients. So either subxiphoid or transhepatic approach can be used without any difference. For this reason, a transhepatic view provides a good alternative when anterior view is not easy to be obtained.

\section{References}

1. Lima A, Bakker J. Noninvasive monitoring of peripheral perfusion. Intensive care medicine. 2005;31(10):1316-26.

2. Boyd JH, Forbes J, Nakada TA, Walley KR, Russell JA. Fluid resuscitation in septic shock: a positive fluid balance and elevated central venous pressure are associated with increased mortality. Critical care medicine. 2011;39(2):259-65.

3. Marik PE, Baram M, Vahid B. Does central venous pressure predict fluid responsiveness?*: A systematic review of the literature and the tale of seven mares. Chest. 2008;134(1):172-8.

4. Sznajder JI, Zveibil FR, Bitterman H, Weiner P, Bursztein S. Central vein catheterization: failure and complication rates by three percutaneous approaches. Archives of internal medicine. 1986;146(2):259-61.

5. Johansson E, Hammarskjöld F, Lundberg D, Arnlind MH. Advantages and disadvantages of peripherally inserted central venous catheters (PICC) compared to other central venous lines: a systematic review of the literature. Acta Oncol.2005 ; 52(5):886-892.

6. Nayeemuddin M, Pherwani AD, Asquith JR. Imaging and management of complications of central venous catheters. Clinical Radiology. 2013;68(5):529-44.

7. Jones AE, Tayal VS, Sullivan DM, Kline JA. Randomized, controlled trial of immediate versus delayed goal-directed ultrasound to identify the cause of nontraumatic hypotension in emergency department patients. Critical care medicine. 2004;32(8):1703-8.

8. Dipti A, Soucy Z, Surana A, Chandra S. Role of inferior vena cava diameter in assessment of volume status: a meta-analysis. The American journal of emergency medicine. 2012;30(8):1414-9.

9. $\mathrm{Ng} \mathrm{L}$, Khine H, Taragin BH, Avner JR, Ushay M, Nunez D. Does bedside sonographic measurement of the inferior vena cava diameter correlate with central venous pressure in the assessment of intravascular volume in children?. Pediatric emergency care. 2013;29(3):337-41. 
10. Kircher BJ, Himelman RB, Schiller NB. Noninvasive estimation of right atrial pressure from the inspiratory collapse of the inferior vena cava. The American journal of cardiology. 1990;66(4):493-6.

11. Yildirimturk O, Tayyareci Y, Erdim R, Ozen E, Yurdakul S, Aytekin V,et al. Assessment of right atrial pressure using echocardiography and correlation with catheterization. Journal of Clinical Ultrasound. 2011;39(6):337-43.

12. Brennan JM, Blair JE, Goonewardena S, Ronan A, Shah D, Vasaiwala S, et al. Reappraisal of the use of inferior vena cava for estimating right atrial pressure. Journal of the American Society of Echocardiography. 2007;20(7):857-61.

13. Ferrada P, Anand RJ, Whelan J, Aboutanos MA, Duane T, Malhotra A,et al. Qualitative assessment of the inferior vena cava: useful tool for the evaluation of fluid status in critically ill patients. The American Surgeon. 2012;78(4):468-70.

14. Miller TE, Gan TJ. Dynamic and Static Parameters of Fluid Responsiveness, Volume 48, Number 1. Anesthesiology: The Journal of the American Society of Anesthesiologists. 2011;114(1):226-.

15. Rudski LG, Lai WW, Afilalo J, Hua L, Handschumacher MD, Chandrasekaran K,et al. Guidelines for the echocardiographic assessment of the right heart in adults: a report from the American Society of Echocardiography: endorsed by the European Association of Echocardiography, a registered branch of the European Society of Cardiology, and the Canadian Society of Echocardiography. Journal of the American Society of Echocardiography. 2010;23(7):685-713.

16. Sawe HR, Haeffele C, Mfinanga JA, Mwafongo VG, Reynolds TA. Predicting Fluid Responsiveness Using Bedside Ultrasound Measurements of the Inferior Vena Cava and Physician Gestalt in the Emergency Department of an Urban Public Hospital in Sub-Saharan Africa. 2016;11(9):e0162772.

17. Michard F. Changes in arterial pressure during mechanical ventilation. Anesthesiology: The Journal of the American Society of Anesthesiologists. 2005;103(2):419-28.

18. Seif D, Mailhot T, Perera P, Mandavia D. Caval Sonography in Shock. Journal of Ultrasound in Medicine. 2012;31(12):188590.

19. Feissel M, Michard F, Faller JP, Teboul JL. The respiratory variation in inferior vena cava diameter as a guide to fluid therapy. Intensive care medicine. 2004;30(9):1834-7.

20. Airapetian N, Maizel J, Alyamani O, Mahjoub $\mathrm{Y}$, Lorne $\mathrm{E}$, Levrard M,et al. Does inferior vena cava respiratory variability predict fluid responsiveness in spontaneously breathing patients?. Critical Care. 2015;19(1):400.

21. Weekes AJ, Tassone HM, Babcock A, Quirke DP, Norton HJ, Jayarama K,et al. Comparison of serial qualitative and quantitative assessments of caval index and left ventricular systolic function during early fluid resuscitation of hypotensive emergency department patients. Academic Emergency Medicine. 2011;18(9):912-21.

22. Lyon M, Blaivas M, Brannam L. Sonographic measurement of the inferior vena cava as a marker of blood loss. The American journal of emergency medicine. 2005;23(1):45-50

23. Moreno FL, Hagan AD, Holmen JR, Pryor TA, Strickland RD, Castle CH. Evaluation of size and dynamics of the inferior vena cava as an index of right-sided cardiac function. American Journal of Cardiology. 1984;53(4):579-85.

24. Panebianco NL, Shofer F, Cheng A, Fischer J, Cody K, Dean AJ. The effect of supine versus upright patient positioning on inferior vena cava metrics. The American journal of emergency medicine. 2014;32(11):1326-9.

25. Hollerbach S, Schultze K, Muscholl M, Schölmerich J. Ultrasonography of the inferior vena cava (IVC) in the diagnosis and monitoring of therapy in patients with chronic congestive heart failure. Deutsche medizinische Wochenschrift (1946). 2001;126(6):129-33.

26. Finnerty NM, Panchal AR, Creagh Boulger AV, Bischof JJ, Amick C, Way DP, et al. Inferior Vena Cava Measurement with Ultrasound: What Is the Best View and Best Mode?. Western Journal of Emergency Medicine. 2017;18(3):496..

27. Charron C, Caille V, Jardin F, Vieillard-Baron A. Echocardiographic measurement of fluid responsiveness. Current opinion in critical care. 2006;12(3):249-54

28. Haydar SA, Moore ET, Higgins GL, Irish CB, Owens WB, Strout TD. Effect of bedside ultrasonography on the certainty of physician clinical decision making for septic patients in the emergency department. Annals of emergency medicine. 2012;60(3):346-58..

29. Kitamura $\mathrm{H}$, Kobayashi C. Impairment of Change in Diameter of the Hepatic Portion of the Inferior Vena Cava. Journal of ultrasound in medicine. 2005;24(3):355-9.

30. Dellinger RP, Levy MM, Rhodes A, Annane D, Gerlach H, Opal SM, et al. Surviving Sepsis Campaign: international guidelines for management of severe sepsis and septic shock, 2012. Intensive care medicine. 2013;39(2):165-228.

31. Michard F, Teboul JL. Predicting fluid responsiveness in ICU patients: a critical analysis of the evidence. Chest. 2002;121(6):2000-8

32. Shala MB, D'CRUZ IA, Johns C, Kaiser J, Clark R. Echocardiography of the inferior vena cava, superior vena cava, and coronary sinus in right heart failure. Echocardiography. 1998;15(8):787- 94

33. Nagueh SF, Kopelen HA, Zoghbi WA. Relation of mean right atrial pressure to echocardiographic and Doppler parameters of right atrial and right ventricular function. Circulation. 1996;93(6):1160-9.

34. Nette RW, le EH, Vletter WB, Krams R, Weimar W, Zietse R. Norepinephrine-induced vasoconstriction results in decreased blood volume in dialysis patients. Nephrology Dialysis Transplantation. 2006;21(5):1305-11.

35. Mintz GS, Kotler MN, Parry WR, Iskandrian AS, Kane SA. Reat- time inferior vena caval ultrasonography: normal and abnormal findings and its use in assessing right-heart function. Circulation. 1981;64(5):1018-25.

36. Zhang Z, Xu X, Ye S, Xu L. Ultrasonographic measurement of the respiratory variation in the inferior vena cava diameter is predictive of fluid responsiveness in critically ill patients: systematic review and meta- analysis. Ultrasound in Medicine and Biology. 2014;40(5):845-53.

37. Jue J, Chung W, Schiller NB. Does inferior vena cava size predict right atrial pressures in patients receiving mechanical ventilation?. Journal of the American Society of Echocardiography. 1992;5(6):613-9.

38. Nagueh SF, Kopelen HA, Zoghbi WA. Relation of mean right atrial pressure to echocardiographic and Doppler parameters of right atrial and right ventricular function. Circulation. 1996;93(6):1160-9.

39. Blehar DJ, Dickman E, Gaspari R. Identification of congestive heart failure via respiratory variation of inferior vena cava diameter. The American journal of emergency medicine. 2009;27(1):71-5.

40. Barbier $\mathrm{C}$, Loubières $\mathrm{Y}$, Schmit $\mathrm{C}$, Hayon J, Ricôme JL, Jardin $\mathrm{F}$, et al. Respiratory changes in inferior vena cava diameter are helpful in predicting fluid responsiveness in ventilated septic patients. Intensive care medicine. 2004:30(9):1740-6.

41. Yanagawa Y, Nishi K, Sakamoto T, Okada Y. Early diagnosis of hypovolemic shock by sonographic measurement of inferior vena cava in trauma patients. Journal of Trauma and Acute Care Surgery. 2005;58(4):825-9.

42. Sefidbakht S, Assadsangabi R, Abbasi HR, Nabavizadeh A. Sonographic measurement of the inferior vena cava as a predictor of shock in trauma patients. Emergency radiology. 2007:14(3):181-5.

43. Akıllı B, Bayır A, Kara F, Ak A, Cander B. Inferior vena cava diameter as a marker of early hemorrhagic shock: a comparative study. Turkish Journal of Trauma and Emergency Surgery. 2010;16(2):113-8.

44. Fields JM, Lee PA, Jenq KY, Mark DG, Panebianco NL, Dean AJ. The interrater reliability of inferior vena cava ultrasound by bedside clinician sonographers in emergency department patients. Academic Emergency Medicine. 2011;18(1):98-101.

45. Akkaya A, Yesilaras M, Aksay E, Sever M, Atilla OD. The interrater reliability of ultrasound imaging of the inferior vena cava performed by emergency residents. The American journal of emergency medicine. 2013;31(10):1509-11.

46. Kulkarni AP, Janarthanan S, Harish MM, Suhail S, Chaudhari H, Agarwal V, et al. Agreement between inferior vena cava diameter measurements by subxiphoid versus transhepatic views. Indian journal of critical care medicine: peer-reviewed, official publication of Indian Society of Critical Care Medicine. 2015;19(12):719.

47. Shah R, Spiegel R, Lu C, Ahmad S. Inferior Vena Cava Flat Ratio as a Predictor of Concordance or Discordance Between Subcostal and Transhepatic Measurements of Collapsibility. Chest. 2016;150(4):293A 